\title{
Fatal Attractors in Parity Games
}

\author{
Michael Huth ${ }^{1}$, Jim Huan-Pu Kuo ${ }^{1}$, and Nir Piterman ${ }^{2}$ \\ 1 Department of Computing, Imperial College London \\ London, SW7 2AZ, United Kingdom \\ \{m.huth, jimhkuo\}@imperial.ac.uk \\ 2 Department of Computer Science, University of Leicester \\ Leicester, LE1 7RH, United Kingdom \\ nir.piterman@leicester.ac.uk
}

\begin{abstract}
We study a new form of attractor in parity games and use it to define solvers that run in PTIME and are partial in that they do not solve all games completely. Technically, for color $c$ this new attractor determines whether player $c \% 2$ can reach a set of nodes $X$ of color $c$ whilst avoiding any nodes of color less than $c$. Such an attractor is fatal if player $c \% 2$ can attract all nodes in $X$ back to $X$ in this manner. Our partial solvers detect fixed-points of nodes based on fatal attractors and correctly classify such nodes as won by player $c \% 2$. Experimental results show that our partial solvers completely solve benchmarks that were constructed to challenge existing full solvers. Our partial solvers also have encouraging run times in practice. For one partial solver we prove that its runtime is in $O\left(|V|^{3}\right)$, that its output game is independent of the order in which attractors are computed, and that it solves all Büchi games 1
\end{abstract}

\section{Introduction}

Parity games are an important foundational structure in formal verification (see e.g. [11]). Mathematically, they can be seen as a representation of the model checking problem for the modal mu-calculus [4, and its exact computational complexity has been an open problem for over twenty years now.

Parity games are infinite, 2-person, 0-sum, graph-based games that are hard to solve. Their nodes, controlled by different players, are colored with natural numbers and the winning condition of plays depends on the minimal color occurring in cycles. The condition for winning a node, therefore, is an alternation of existential and universal quantification. In practice, this means that the maximal color of its coloring function is the only exponential source for the worst-case complexity of most parity game solvers, e.g. for those in [11810].

Research on solving parity games may be loosely grouped into the following approaches: design of algorithms that solve all parity games by construction and that so far all have exponential or sub-exponential worst-case complexity

\footnotetext{
${ }^{1}$ A preliminary version of the results reported in this paper was presented at the GAMES 2012 workshop in Naples, Italy, on 11 September 2012.
} 
(e.g. 118109$]$ ), restriction of parity games to classes for which polynomialtime algorithms can be devised as complete solvers (e.g. [13]), and practical improvements to solvers so that they perform well across benchmarks (e.g. [5]).

We here propose a new approach that relates to, and potentially impacts, all of these aforementioned activities. We want to design and evaluate a new form of "partial" parity game solver. These are solvers that are well defined for all parity games but that may not solve all games completely, i.e. for some parity games they may not decide the winning status of some nodes. For us, a partial solver has an arbitrary parity game as input and returns two things: a sub-game of the input game, and a classification of the winning status of all nodes of the input game that are not in that sub-game. In particular, the returned sub-game is empty if, and only if, the partial solver classified the winners for all input nodes.

The input/output type of our partial solvers clearly relates them to so called preprocessors that may decide the winner of nodes whose structure makes such a decision an easy static criterion (e.g. in the elimination of self-loops or dead ends [5]). But we here search for dynamic criteria that allow partial solvers to completely solve a range of benchmarks of parity games. This ambition sets our work apart from research on preprocessors but is consistent with it as one can always run a partial solver as preprocessor.

The motivation for the study reported in this paper is that we want to investigate what theoretical building blocks one may create and use for designing partial solvers that run in polynomial time and work well on many games, whether partial solvers can be components of more efficient complete solvers, and whether there are interesting subclasses of parity games for which partial solvers completely solve all games. In particular, one may study the class of output games of a PTIME partial solver in lieu of studying the aforementioned open problem for all parity games.

We summarize the main contributions made in this paper:

- We present a new form of attractor that can be used in fixed-point computations to detect winning nodes for a given player in parity games.

- We propose several designs of partial solvers for parity games by using this new attractor within fixed-point computations.

- We analyze these partial solvers and show, e.g., that they work in PTIME and that one of them is independent of the order of attractor computation.

- And we evaluate these partial solvers against known benchmarks and report that these experiments have very encouraging results.

Outline of paper. Section 2 contains needed formal background and fixes notation. Section 3 introduces the building block of our partial solvers, a new form of attractor. Some partial solvers based on this attractor are presented in Section 4 theoretical results about these partial solvers are proved in Section [5] and experimental results for these partial solvers run on benchmarks are reported and discussed in Section 6. We summarize and conclude the paper in Section 7. 


\section{Preliminaries}

We write $\mathbb{N}$ for the set $\{0,1, \ldots\}$ of natural numbers. A parity game $G$ is a tuple $\left(V, V_{0}, V_{1}, E, c\right)$, where $V$ is a set of nodes partitioned into possibly empty node sets $V_{0}$ and $V_{1}$, with an edge relation $E \subseteq V \times V$ (where for all $v$ in $V$ there is a $w$ in $V$ with $(v, w)$ in $E)$, and a coloring function $c: V \rightarrow \mathbb{N}$. In figures, $c(v)$ is written within nodes $v$, nodes in $V_{0}$ are depicted as circles and nodes in $V_{1}$ as squares. For $v$ in $V$, we write $v$. $E$ for node set $\{w \in V \mid(v, w) \in E\}$ of successors of $v$. By abuse of language, we call a subset $U$ of $V$ a sub-game of $G$ if the game graph $(U, E \cap(U \times U))$ is such that all nodes in $U$ have some successor. We write $\mathcal{P} G$ for the class of all finite parity games $G$, which includes the parity game with empty node set for our convenience. We only consider games in $\mathcal{P} G$.

Throughout, we write $p$ for one of 0 or 1 and $1-p$ for the other player. In a parity game, player $p$ owns the nodes in $V_{p}$. A play from some node $v_{0}$ results in an infinite play $r=v_{0} v_{1} \ldots$ in $(V, E)$ where the player who owns $v_{i}$ chooses the successor $v_{i+1}$ such that $\left(v_{i}, v_{i+1}\right)$ is in $E$. Let $\operatorname{Inf}(r)$ be the set of colors that occur in $r$ infinitely often: $\operatorname{lnf}(r)=\left\{k \in \mathbb{N} \mid \forall j \in \mathbb{N}: \exists i \in \mathbb{N}: i>j\right.$ and $\left.k=c\left(v_{i}\right)\right\}$. Player 0 wins play $r$ iff $\min \operatorname{lnf}(P)$ is even; otherwise player 1 wins play $r$.

A strategy for player $p$ is a total function $\tau: V_{p} \rightarrow V$ such that $(v, \tau(v))$ is in $E$ for all $v \in V_{p}$. A play $r$ is consistent with $\tau$ if each node $v_{i}$ in $r$ owned by player $p$ satisfies $v_{i+1}=\tau\left(v_{i}\right)$. It is well known that each parity game is determined: node set $V$ is the disjoint union of two, possibly empty, sets $W_{0}$ and $W_{1}$, the winning regions of players 0 and 1 (respectively). Moreover, strategies $\sigma: V_{0} \rightarrow V$ and $\pi: V_{1} \rightarrow V$ can be computed such that

- all plays beginning in $W_{0}$ and consistent with $\sigma$ are won by player 0 ; and

- all plays beginning in $W_{1}$ and consistent with $\pi$ are won by player 1 .

Solving a parity game means computing such data $\left(W_{0}, W_{1}, \sigma, \pi\right)$.

Example 1. In the parity game $G$ depicted in Figure 1 the winning regions are $W_{1}=\left\{v_{3}, v_{5}, v_{7}\right\}$ and $W_{0}=\left\{v_{0}, v_{1}, v_{2}, v_{4}, v_{6}, v_{8}, v_{9}, v_{10}, v_{11}\right\}$. Let $\sigma$ move from $v_{2}$ to $v_{4}$, from $v_{6}$ to $v_{8}$, from $v_{9}$ to $v_{8}$, and from $v_{10}$ to $v_{9}$. Then $\sigma$ is a winning strategy for player 0 on $W_{0}$. And every strategy $\pi$ is winning for player 1 on $W_{1}$.

\section{Fatal Attractors}

In this section we define a special type of attractor that is used for our partial solvers in the next section. We start by recalling the normal definition of attractor, and that of a trap, and then generalize the former to our purposes.

Definition 1. Let $X$ be a node set in parity game $G$. For player $p$ in $\{0,1\}$, set

$$
\begin{aligned}
\operatorname{cpre}_{p}(X) & =\left\{v \in V_{p} \mid v \cdot E \cap X \neq \emptyset\right\} \cup\left\{v \in V_{1-p} \mid v \cdot E \subseteq X\right\} \\
\operatorname{Attr}_{p}[G, X] & =\mu Z .\left(X \cup \operatorname{cpre}_{p}(Z)\right)
\end{aligned}
$$

where $\mu Z . F(Z)$ denotes the least fixed point of a monotone function $F: 2^{V} \rightarrow 2^{V}$.

The control predecessor of a node set $X$ for $p$ in (11) is the set of nodes from which player $p$ can force to get to $X$ in exactly one move. The attractor for 
player $p$ to a set $X$ in (2) is computed via a least fixed-point as the set of nodes from which player $p$ can force the game in zero or more moves to get to the set $X$. Dually, a trap for player $p$ is a region from which player $p$ cannot escape.

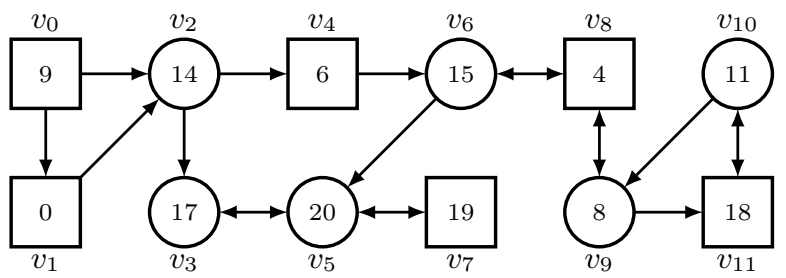

Fig. 1. A parity game: circles denote nodes in $V_{0}$, squares denote nodes in $V_{1}$

Definition 2. Node set $X$ in parity game $G$ is a trap for player $p$ ( $p$-trap) if for all $v \in V_{p} \cap X$ we have $v . E \subseteq X$ and for all $v \in V_{1-p} \cap X$ we have $v \cdot E \cap X \neq \emptyset$.

It is well known that the complement of an attractor for player $p$ is a $p$-trap and that it is a sub-game. We state this here formally as a reference:

Theorem 1. Given a node set $X$ in a parity game $G$, the set $V \backslash \operatorname{Attr}_{p}[G, X]$ is a p-trap and a sub-game of $G$.

We now define a new type of attractor, which will be a crucial ingredient in the definition of all our partial solvers.

Definition 3. Let $A$ and $X$ be node sets in parity game $G$, let $p$ in $\{0,1\}$ be $a$ player, and $c$ a color in $G$. We set

$$
\begin{aligned}
\operatorname{mpre}_{p}(A, X, c)= & \left\{v \in V_{p} \mid c(v) \geq c \wedge v \cdot E \cap(A \cup X) \neq \emptyset\right\} \cup \\
& \left\{v \in V_{1-p} \mid c(v) \geq c \wedge v \cdot E \subseteq A \cup X\right\} \\
\operatorname{MAttr}_{p}(X, c)= & \mu Z \cdot \operatorname{mpre}_{p}(Z, X, c)
\end{aligned}
$$

The monotone control predecessor mpre $\operatorname{mot}_{p}(A, X, c)$ of node set $A$ for $p$ with target $X$ is the set of nodes of color at least $c$ from which player $p$ can force to get to either $A$ or $X$ in one move. The monotone attractor $\operatorname{MAttr}_{p}(X, c)$ for $p$ with target $X$ is the set of nodes from which player $p$ can force the game in one or more moves to $X$ by only meeting nodes whose color is at least $c$. Notice that the target set $X$ is kept external to the attractor. Thus, if some node $x$ in $X$ is included in $\operatorname{MAttr}_{p}(X, c)$ it is so as it is attracted to $X$ in at least one step.

Our control predecessor and attractor are different from the "normal" ones in a few ways. First, ours take into account the color $c$ as a formal parameter. They add only nodes that have color at least $c$. Second, as discussed above, the target set $X$ itself is not included in the computation by default. For example, $\operatorname{MAttr}_{p}(X, c)$ includes states from $X$ only if they can be attracted to $X$. 
We now show the main usage of this new operator by studying how specific instantiations thereof can compute so called fatal attractors.

Definition 4. Let $X$ be a set of nodes of color $c$, where $p=c \% 2$.

1. For such an $X$ we denote $p$ by $p(X)$ and $c$ by $c(X)$. We denote $\operatorname{MAttr}_{p}(X, c)$ by $\operatorname{MA}(X)$. If $X=\{x\}$ is a singleton, we denote $\operatorname{MA}(X)$ by $\operatorname{MA}(x)$.

2. We say that $\mathrm{MA}(X)$ is a fatal attractor if $X \subseteq \mathrm{MA}(X)$.

We note that fatal attractors $\mathrm{MA}(X)$ are node sets that are won by player $p(X)$ in $G$. The winning strategy is the attractor strategy corresponding to the least fixed-point computation in $\operatorname{MAttr}_{p}(X, c)$. First of all, player $p(X)$ can force, from all nodes in $\mathrm{MA}(X)$, to reach some node in $X$ in at least one move. Then, player $p(X)$ can do this again from this node in $X$ as $X$ is a subset of $\operatorname{MA}(X)$. At the same time, by definition of $\operatorname{MAttr}_{p}(X, c)$ and $\operatorname{mpre}_{p}(A, X, c)$, the attraction ensures that only colors of value at least $c$ are encountered. So in plays starting in $\mathrm{MA}(X)$ and consistent with that strategy, every visit to a node of parity $1-p(X)$ is followed later by a visit to a node of color $c(X)$. It follows that in an infinite play consistent with this strategy and starting in $\mathrm{MA}(X)$, the minimal color to be visited infinitely often is $c$ - which is of $p$ 's parity.

Theorem 2. Let $\mathrm{MA}(X)$ be fatal in parity game $G$. Then the attractor strategy for player $p(X)$ on $\mathrm{MA}(X)$ is winning for $p(X)$ on $\mathrm{MA}(X)$ in $G$.

Let us consider the case when $X$ is a singleton $\{k\}$ and $\operatorname{MA}(k)$ is not fatal. Suppose that there is an edge $(k, w)$ in $E$ with $w$ in $\operatorname{MA}(k)$. We show that this edge cannot be part of a winning strategy (of either player) in $G$. Since MA $(k)$ is not fatal, $k$ must be in $V_{1-p(k)}$ and so is controlled by player $1-p(k)$. But if that player were to move from $k$ to $w$ in a memoryless strategy, player $p(k)$ could then attract the play from $w$ back to $k$ without visiting colors of parity $1-p(k)$ and smaller than $c(k)$, since $w$ is in $\operatorname{MA}(k)$. And, by the existence of memoryless winning strategies [4], this would ensure that the play is won by player $p(k)$ as the minimal infinitely occurring color would have parity $p(k)$. We summarize:

Lemma 1. Let $\mathrm{MA}(k)$ be not fatal for node $k$. Then we may remove edge $(k, w)$ in $E$ if $w$ is in $\mathrm{MA}(k)$, without changing winning regions of parity game $G$.

Example 2. For $G$ in Figure 1 the only colors $k$ for which $\mathrm{MA}(k)$ is fatal are 4 and 8: $\mathrm{MA}(4)$ equals $\left\{v_{2}, v_{4}, v_{6}, v_{8}, v_{9}, v_{10}, v_{11}\right\}$ and $\mathrm{MA}(8)$ equals $\left\{v_{9}, v_{10}, v_{11}\right\}$. In particular, $\mathrm{MA}(8)$ is contained in $\mathrm{MA}(4)$ and nodes $v_{1}$ and $v_{0}$ are attracted to $\mathrm{MA}(4)$ in $G$ by player 0 . And $v_{11}$ is in $\mathrm{MA}(11)$ (but the node of color $11, v_{10}$, is not), so edge $\left(v_{10}, v_{11}\right)$ may be removed.

\section{Partial Solvers}

We can use the above definitions and results to define partial solvers next. Their soundness will be shown in Section 5. 


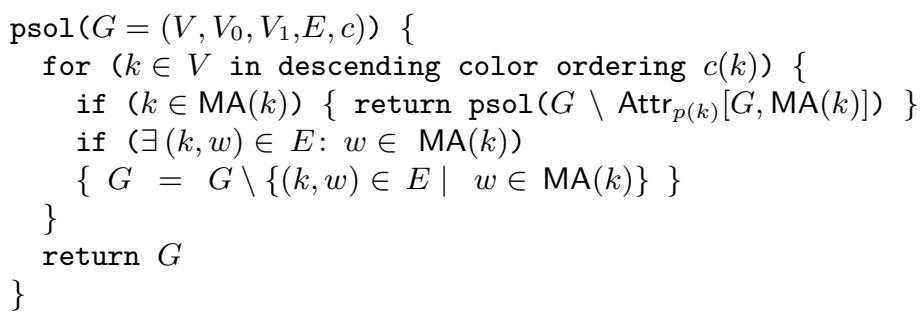

Fig. 2. Partial solver psol based on detection of fatal attractors $\mathrm{MA}(k)$ and fatal moves

\subsection{Partial Solver psol}

Figure 2 shows the pseudocode of a partial solver, named psol, based on $\operatorname{MA}(X)$ for singleton sets $X$. Solver psol explores the parity game $G$ in descending color ordering. For each node $k$, it constructs $\mathrm{MA}(k)$, and aims to do one of two things:

- If node $k$ is in $\mathrm{MA}(k)$, then $\mathrm{MA}(k)$ is fatal for player $1-p(k)$, thus node set $\operatorname{Attr}_{p(k)}[G, \mathrm{MA}(k)]$ is a winning region of player $p(k)$, and removed from $G$.

- If node $k$ is not in $\operatorname{MA}(k)$, and there is a $(k, w)$ in $E$ where $w$ is in $\operatorname{MA}(k)$, all such edges $(k, w)$ are removed from $E$ and the iteration continues.

If for no $k$ in $V$ attractor $\mathrm{MA}(k)$ is fatal, game $G$ is returned as is - empty if psol solves $G$ completely. The accumulation of winning regions and computation of winning strategies are omitted from the pseudocode for improved readability.

Example 3. In a run of psol on $G$ from Figure 11 there is no effect for colors larger than 11. For $c=11$, psol removes edge $\left(v_{10}, v_{11}\right)$ as $v_{11}$ is in $\mathrm{MA}(11)$. The next effect is for $c=8$, when the fatal attractor $\operatorname{MA}(8)=\left\{v_{9}, v_{10}, v_{11}\right\}$ is detected and removed from $G$ (the previous edge removal did not cause the attractor to be fatal). On the remaining game, the next effect occurs when $c=4$, and when the fatal attractor $\mathrm{MA}(4)$ is $\left\{v_{2}, v_{4}, v_{6}, v_{8}\right\}$ in that remaining game. As player 0 can attract $v_{0}$ and $v_{1}$ to this as well, all these nodes are removed and the remaining game has node set $\left\{v_{3}, v_{5}, v_{7}\right\}$. As there is no more effect of psol on that remaining game, it is returned as the output of psol's run.

\subsection{Partial Solver psolB}

Figure 3 shows the pseudocode of another partial solver, named psolB (the "B" suggests a relationship to "Büchi"), based on $\mathrm{MA}(X)$, where $X$ is a set of nodes of the same color. This time, the operator $\operatorname{MA}(X)$ is used within a greatest fixed-point in order to discover the largest set of nodes of a certain color that can be (fatally) attracted to itself. Accordingly, the greatest fixed-point starts from all the nodes of a certain color and gradually removes those that cannot be attracted to the same color. When the fixed-point stabilizes, it includes the set of nodes of the given color that can be (fatally) attracted to itself. This node set can be removed (as a winning region for player $d \% 2$ ) and the residual game analyzed recursively. As before, the colors are explored in descending order. 


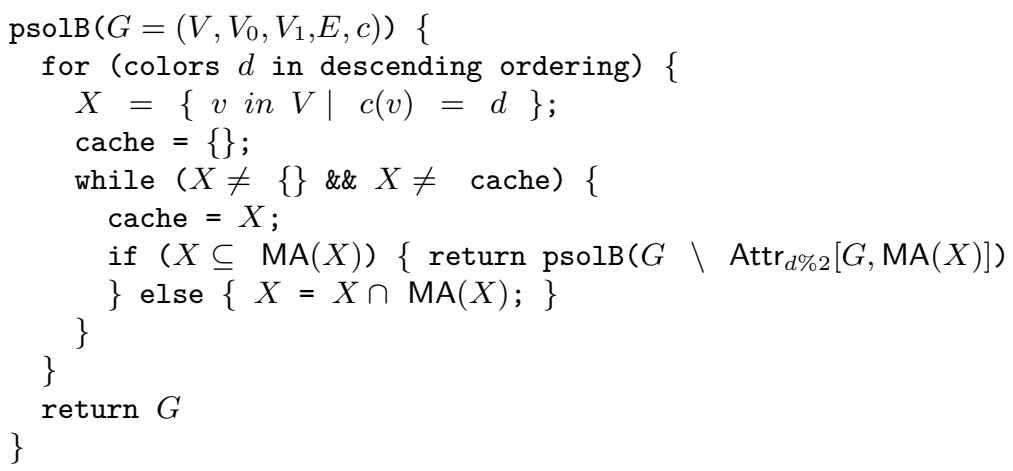

Fig. 3. Partial solver psolB

We make two observations. First, if we were to replace the recursive calls in psolB with the removal of the winning region from $G$ and a continuation of the iteration, we would get an implementation that discovers less fatal attractors. Second, edge removal in psol relies on the set $X$ being a singleton. A similar removal could be achieved in psolB when the size of $X$ is reduced by one (in the operation $X=X \cap \mathrm{MA}(X)$ ). Indeed, in such a case the removed node would not be removed and the current value of $X$ be realized as fatal. We have not tested this edge removal approach experimentally for this variant of psolB.

Example 4. A run of psolB on $G$ from Figure 1 has the same effect as the one for psol, except that psolB does not remove edge $\left(v_{10}, v_{11}\right)$ when $c=11$.

A way of comparing partial solvers $P_{1}$ and $P_{2}$ is to say that $P_{1} \leq P_{2}$ if, and only if, for all parity games $G$ the set of nodes in the output sub-game $P_{1}(G)$ is a subset of the set of nodes of the output sub-game $P_{2}(G)$. We note that psol and psolB are incomparable for this intensional pre-order over partial solvers.

\subsection{Partial Solver psolQ}

It seems that psolB is more general than psol in that if there is a singleton $X$ with $X \subseteq \mathrm{MA}(X)$ then psolB will discover this as well. However, the requirement to attract to a single node seems too strong. Solver psolB removes this restriction and allows to attract to more than one node, albeit of the same color. Now we design a partial solver psolQ that can attract to a set of nodes of more than one color (the "Q" is our code name for this "Q" uantified layer of colors of the same parity). Solver psolQ allows to combine attraction to multiple colors by adding them gradually and taking care to "fix" visits to nodes of opposite parity.

We extend the definition of mpre and MAttr to allow inclusion of more (safe) nodes when collecting nodes in the attractor. 


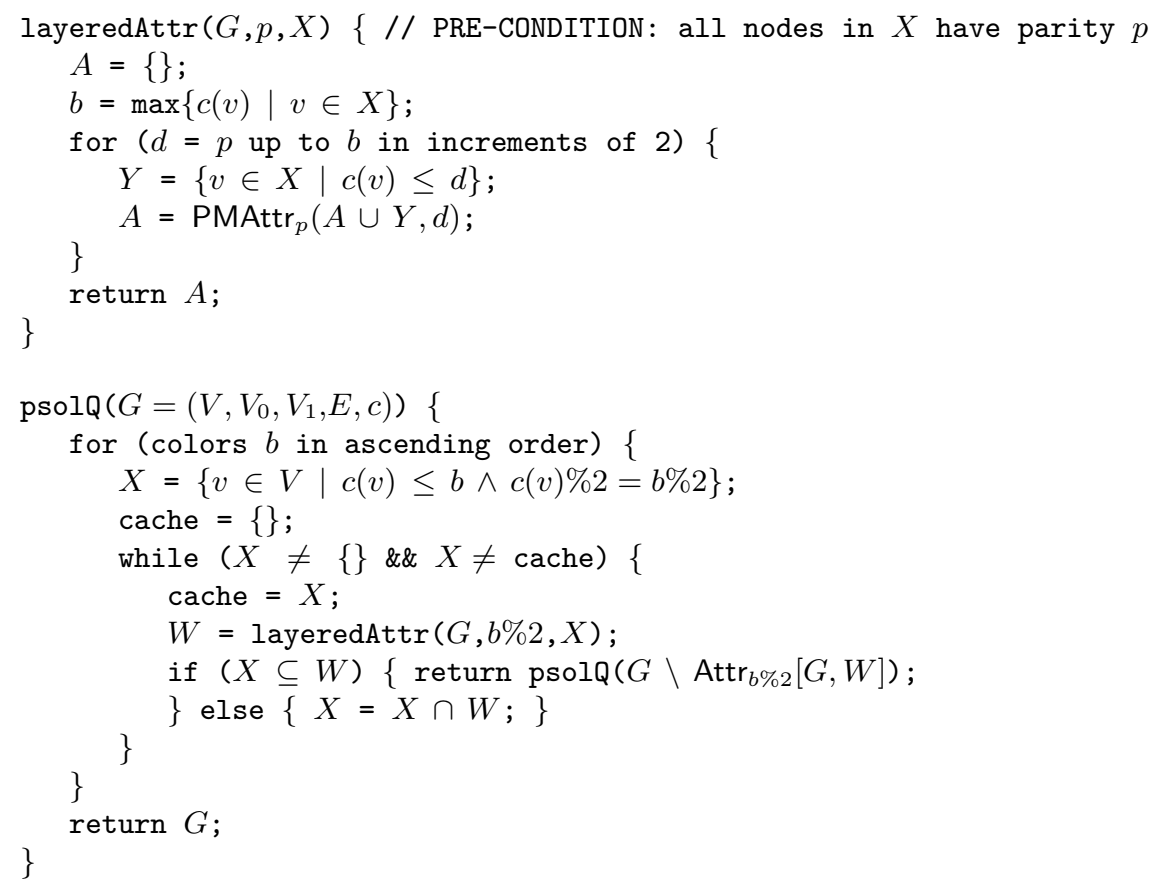

Fig. 4. Operator layeredAttr $(G, p, X)$ and partial solver psolQ

Definition 5. Let $A$ and $X$ be node sets in parity game $G$, let $p$ in $\{0,1\}$ be $a$ player, and $c$ a color in $G$. We set

$$
\begin{aligned}
\operatorname{pmpre}_{p}(A, X, c)= & \left\{v \in V_{p} \mid(c(v) \geq c \vee v \in X) \wedge v \cdot E \cap(A \cup X) \neq \emptyset\right\} \cup \\
& \left\{v \in V_{1-p} \mid(c(v) \geq c \vee v \in X) \wedge v \cdot E \subseteq A \cup X\right\} \\
\operatorname{PMAttr}_{p}(X, c)= & \mu Z \cdot \operatorname{pmpre}_{p}(Z, X, c)
\end{aligned}
$$

The permissive monotone predecessor in (4) adds to the monotone predecessor also nodes that are in $X$ itself even if their color is lower than $c$, i.e., they violate the monotonicity requirement. The permissive monotone attractor in (5) then uses the permissive predecessor instead of the simpler predecessor. This is used for two purposes. First, when the set $X$ includes nodes of multiple colors some of them lower than $c$. Then, inclusion of nodes from $X$ does not destroy the properties of fatal attraction. Second, increasing the set $X$ of target nodes allows us to include the previous target and the attractor to it as set of "permissible" nodes. This creates a layered structure of attractors.

We use the permissive attractor to define psolQ. Figure 4 presents the pseudo code of operator layeredAttr $(G, p, X)$. It is an attractor that combines attraction to nodes of multiple color. It takes a set $X$ of colors of the same parity $p$. It considers increasing subsets of $X$ with more and more colors and tries to attract fatally to them. It starts from a set $Y_{p}$ of nodes of parity $p$ with color $p$ and computes $\mathrm{MA}\left(Y_{p}\right)$. At this stage, the difference between pmpre and mpre does not apply as $Y_{p}$ contains nodes of only one color and $A$ is empty. Then, instead of 
stopping as before, it continues to accumulate more nodes. It creates the set $Y_{p+2}$ of the nodes of parity $p$ with color $p$ or $p+2$. Then, $\operatorname{PMAttr}_{p}\left(A \cup Y_{p+2}, p+2\right)$ includes all the previous nodes in $A$ (as all nodes in $A$ are now permissible) and all nodes that can be attracted to them or to $Y_{p+2}$ through nodes of color at least $p+2$. This way, even if nodes of a color lower than $p+2$ are included they will be ensured to be either in the previous attractor or of the right parity. Then $Y$ is increased again to include some more nodes of $p$ 's parity. This process continues until it includes all nodes in $X$.

This layered attractor may also be fatal:

Definition 6. We say that layeredAttr $(G, p, X)$ is fatal if $X$ is a subset of layeredAttr $(G, p, X)$.

As before, fatal layered attractors are won by player $p$ in $G$. The winning strategy is more complicated as it has to take into account the number of iteration in the for loop in which a node was first discovered. Every node in layeredAttr $(G, p, X)$ belongs to a layer corresponding to a maximal color $d$. From a node in layer $d$, player $p$ can force to reach some node in $Y_{d} \subseteq X$ or some node in a lower layer $d^{\prime}$. As the number of layers is finite, eventually some node in $X$ is reached. When reaching $X$, player $p$ can attract to $X$ in the same layered fashion again as $X$ is a subset of layeredAttr $(G, p, X)$. Along the way, while attracting through layer $d$ we are ensured that only colors at least $d$ or of a lower layer are encountered. So in plays starting in layeredAttr $(G, p, X)$ and consistent with that strategy, every visit to a node of parity $1-p$ is followed later by a visit to a node of parity $p$ of lower color.

Theorem 3. Let layeredAttr $(G, p, X)$ be fatal in parity game $G$. Then the layered attractor strategy for player $p$ on layeredAttr $(G, p, X)$ is winning for $p$ on layeredAttr $(G, p, X)$ in $G$.

Pseudo code of solver psolQ is also shown in Figure 4 psolQ prepares increasing sets of nodes $X$ of the same color and calls layeredAttr within a greatest fixedpoint. For a set $X$, the greatest fixed-point attempts to discover the largest set of nodes within $X$ that can be fatally attracted to itself (in a layered fashion). Accordingly, the greatest fixed-point starts from all the nodes in $X$ and gradually removes those that cannot be attracted to $X$. When the fixed-point stabilizes, it includes a set of nodes of the same parity that can be attracted to itself. These are removed (along with the normal attractor to them) and the residual game is analyzed recursively.

We note that the first two iterations of psolQ are equivalent to calling psolB on colors 0 and 1 . Then, every iteration of psolQ extends the number of colors considered. In particular, in the last two iterations of psolQ the value of $b$ is the maximal possible value of the appropriate parity. It follows that the sets $X$ defined in these last two iterations include all nodes of the given parity. These last two computations of greatest fixed-points are the most general and subsume all previous greatest fixed-point computations. We discuss in Section 6 why we increase the bound $b$ gradually and do not consider these two iterations alone. 
Example 5. The run of psolQ on $G$ from Figure 1 finds a fatal attractor for bound $b=4$, which removes all nodes except $v_{3}, v_{5}$, and $v_{7}$. For $b=19$, it realizes that these nodes are won by player 1 , and outputs the empty game. That psolQ is a partial solver can be seen in Figure [5] which depicts a game that is not modified at all by psolQ and so is returned as is.

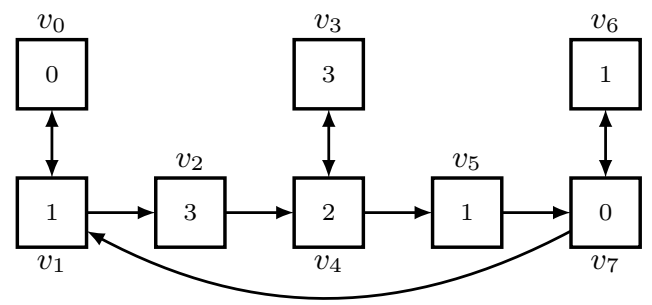

Fig. 5. A 1-player parity game modified by neither psol, psolB nor psolQ

\section{$5 \quad$ Properties of Our Partial Solvers}

We now discuss the properties of our partial solvers, looking first at their soundness and computational complexity.

\subsection{Soundness and Computational Complexity}

Theorem 4. 1. The partial solvers $\mathrm{psol}$, psolB, and $\mathrm{psolQ}$ are sound.

2. The running time for psol and psolB is in $O\left(|V|^{2} \cdot|E|\right)$.

3. And psol and psolB can be implemented to run in time $O\left(|V|^{3}\right)$.

4. And psolQ runs in time $O\left(|V|^{2} \cdot|E| \cdot|c|\right)$ with $|c|$ the number of colors in $G$.

If $\mathrm{psolQ}$ were to restrict attention to the last two iterations of the for loop, i.e., those that compute the greatest fixed-point with the maximal even color and the maximal odd color, the run time of psolQ would be bounded by $O\left(|V|^{2} \cdot|E|\right)$. For such a version of psolQ we also ran experiments on our benchmarks and do not report these results, except to say that this version performs considerably worse than psolQ in practice. We believe that this is so since psolQ more quickly discovers small winning regions that "destabilize" the rest of the games.

\subsection{Robustness of psolB}

Our pseudo-code for psolB iterates through colors in descending order. A natural question is whether the computed output game depends on the order in which these colors are iterated. Below, we formally state that the outcome of psolB is indeed independent of the iteration order. This suggests that these solvers are a form of polynomial-time projection of parity games onto sub-games. 
Let us formalize this. Let $\pi$ be some sequence of colors in $G$, that may omit or repeat some colors from $G$. Let $\operatorname{psolB}(\pi)$ be a version of psolB that checks for (and removes) fatal attractors according to the order in $\pi$ (including any color repetitions in $\pi$ ). We say that $\operatorname{psolB}(\pi)$ is stable if for every color $c_{1}$, the input/output behavior of $\operatorname{psolB}(\pi)$ and $\operatorname{psolB}\left(\pi \cdot c_{1}\right)$ are the same. That is, the sequence $\pi$ leads psolB to stabilization in the sense that every extension of the version $\operatorname{psolB}(\pi)$ with one color does not change the input/output behavior.

Theorem 5. Let $\pi_{1}$ and $\pi_{2}$ be sequences of colors with $\operatorname{psolB}\left(\pi_{1}\right)$ and $\operatorname{psolB}\left(\pi_{2}\right)$ stable. Then $G_{1}$ equals $G_{2}$ if $G_{i}$ is the output of $\operatorname{psolB}\left(\pi_{i}\right)$ on $G$, for $1 \leq i \leq 2$.

Next, we formally define classes of parity games, those that psolB solves completely and those that psolB does not modify.

Definition 7. We define class $\mathcal{S}$ (for "Solved") to consist of those parity games $G$ for which $\operatorname{psolB}(G)$ outputs the empty game. And we define $\mathcal{K}$ (for "Kernel") as the class of those parity games $G$ for which $\operatorname{psolB}(G)$ outputs $G$ again.

The meaning of psolB is therefore a total, idempotent function of type $\mathcal{P} G \rightarrow \mathcal{K}$ that has $\mathcal{S}$ as inverse image of the empty parity game. By virtue of Theorem 5 classes $\mathcal{S}$ and $\mathcal{K}$ are semantic in nature.

We now show that $\mathcal{S}$ contains the class of Büchi games, which we identify with parity games $G$ with color 0 and 1 and where nodes with color 0 are those that player 0 wants to reach infinitely often.

Theorem 6. Let $G$ be a parity game whose colors are only 0 and 1 . Then $G$ is in $\mathcal{S}$, i.e. psolB completely solves $G$.

We point out that $\mathcal{S}$ does not contain some game types for which polynomialtime solvers are known. For example, not all 1-player parity games are in $\mathcal{S}$ (see Figure 5 ). Class $\mathcal{S}$ is also not closed under sub-games.

\section{Experimental Results}

\subsection{Experimental Setup}

We wrote Scala implementations of psol, psolB, and psolQ, and of Zielonka's solver (zlka) that rely on the same data structures and do not compute winning strategies - which has routine administrative overhead. The (parity) Game object has a map of Nodes (objects) with node identifiers (integers) as the keys. Apart from colors and owner type (0 or 1), each Node has two lists of identifiers, one for successors and one for predecessors in the game graph $(V, E)$. For attractor computation, the predecessor list is used to perform "backward" attraction.

This uniform use of data types allows for a first informed comparison. We chose $z l k a$ as a reference implementation since it seems to work well in practice on many games [5]. We then compared the performance of these implementations on all eight non-random, structured game types produced by the PGSolver tool 6]. Here is a list of brief descriptions of these game types. 
- Clique: fully connected games with alternating colors and no self-loops.

- Ladder: layers of node pairs with connections between adjacent layers.

- Recursive Ladder: layers of 5-node blocks with loops.

- Strategy Impr: worst cases for strategy improvement solvers.

- Model Checker Ladder: layers of 4-node blocks.

- Tower Of Hanoi: captures well-known puzzle.

- Elevator Verification: a verification problem for an elevator model.

- Jurdzinski: worst cases for small progress measure solvers.

The first seven types take as game parameter a natural number $n$ as input, whereas Jurdzinski takes a pair of such numbers $n, m$ as game parameter.

For regression testing, we verified for all tested games that the winning regions of psol, psolB, psolQ and zlka are consistent with those computed by PGSolver. Runs of these algorithms that took longer than 20 minutes (i.e. 1200K milliseconds) or for which the machine exhausted the available memory during solver computation are recorded as aborts ("abo") - the most frequent reason for abo was that the used machine ran out of memory. All experiments were conducted on the same machine with an Intel ${ }^{\circledR}$ Core $^{\mathrm{TM}}$ i5 (four cores) CPU at $3.20 \mathrm{GHz}$ and $8 \mathrm{G}$ of RAM, running on a Ubuntu 11.04 Linux operating system.

For most game types, we used unbounded binary search starting with 2 and then iteratively doubling that value, in order to determine the abo boundary value for parameter $n$ within an accuracy of plus/minus 10. As the game type Jurdzinski $[n, m]$ has two parameters, we conducted three unbounded binary searches here: one where $n$ is fixed at 10, another where $m$ is fixed at 10 , and a third one where $n$ equals $m$. We used a larger parameter configuration $(10 \times$ power of two) for Jurdzinski games.

We report here only the last two powers of two for which one of the partial solvers didn't timeout, as well as the boundary values for each solver. For game types whose boundary value was less than 10 (Tower Of Hanoi and Elevator Verification), we didn't use binary search but incremented $n$ by 1 . Finally, if a partial solver didn't solve its input game completely, we ran zlka on the remaining game and added the observed running times for zlka to that of the partial solver. (This occurred for Elevator Verification for psol and psolB.)

\subsection{Experiments on Structured Games}

Our experimental results are depicted in Figures 6 and 17, colored green (respectively red) for the partial solver with best (respectively worst) result. Running times are reported in milliseconds. The most important outcome is that partial solvers psol and psolB solved seven of the eight game types completely for all runs that did not time out, the exception being Elevator Verification; and that psolQ solved all eight game types completely. This suggests that partial solvers can actually be used as solvers on a range of structured game types.

We now compare the performance of these partial solvers and of zlka. There were ten experiments, three for Jurdzinski and one for each of the remaining seven game types. For seven out of these ten experiments, psolB had the largest boundary value of the parameter and so seems to perform best overall. The solver 
Clique $[n]$

\begin{tabular}{|r||r|r|r|r|}
\hline$n$ & psol & psolB & psolQ & zlka \\
\hline \hline $2^{* * 11}$ & 6016.68 & 48691.72 & 3281.57 & 12862.92 \\
\hline $2^{* * 12}$ & abo & 164126.06 & 28122.96 & 76427.44 \\
\hline \hline $20 \mathrm{~min}$ & $n=3680$ & $n=5232$ & $n=4608$ & $n=5104$ \\
\hline
\end{tabular}

Ladder $[n]$

\begin{tabular}{|r||r|r|r|r|}
\hline$n$ & psol & psolB & psolQ & zlka \\
\hline \hline $2^{* *} 19$ & abo & 22440.57 & 26759.85 & 24406.79 \\
\hline $2^{* *} 20$ & abo & 47139.96 & 59238.77 & 75270.74 \\
\hline \hline $20 m i n$ & $n=14712$ & $n=1596624$ & $n=1415776$ & $n=1242376$ \\
\hline
\end{tabular}

Model Checker Ladder $[n]$

\begin{tabular}{|r||r|r|r|r|}
\hline$n$ & psol & psolB & psolQ & zlka \\
\hline \hline $2^{* *} 12$ & 119291.99 & 90366.80 & 117006.17 & 79284.72 \\
\hline $2^{* *} 13$ & 560002.68 & 457049.22 & 644225.37 & 398592.74 \\
\hline \hline $20 \mathrm{~min}$ & $n=11528$ & $n=12288$ & $n=10928$ & $n=13248$ \\
\hline
\end{tabular}

Recursive Ladder $[n]$

\begin{tabular}{|r||r|r|r|r|}
\hline$n$ & psol & psolB & psolQ & zlka \\
\hline \hline $2^{* *} 12$ & abo & abo & 138956.08 & abo \\
\hline $2^{* *} 13$ & abo & abo & 606868.31 & abo \\
\hline \hline $20 \mathrm{~min}$ & $n=1560$ & $n=2064$ & $n=11352$ & $n=32$ \\
\hline
\end{tabular}

Strategy $\operatorname{Impr}[n]$

\begin{tabular}{|r||r|r|r|r|}
\hline$n$ & psol & psolB & psolQ & zlka \\
\hline \hline $2^{* * 10}$ & 174913.85 & 134795.46 & abo & abo \\
\hline $2^{* *} 11$ & 909401.03 & 631963.68 & abo & abo \\
\hline \hline $20 \mathrm{~min}$ & $n=2368$ & $n=2672$ & $n=40$ & $n=24$ \\
\hline
\end{tabular}

Tower Of Hanoi $[n]$

\begin{tabular}{|r||r|r|r|r|}
\hline$n$ & psol & psolB & psolQ & zlka \\
\hline \hline 9 & 272095.32 & 54543.31 & 610264.18 & 56780.41 \\
\hline 10 & abo & 397728.33 & abo & 390407.41 \\
\hline \hline $20 \mathrm{~min}$ & $n=9$ & $n=10$ & $n=9$ & $n=10$ \\
\hline
\end{tabular}

Elevator Verification $[n]$

\begin{tabular}{|r||r|r|r|r|}
\hline$n$ & psol & psolb & psolQ & zlka \\
\hline \hline 1 & 171.63 & 120.59 & 147.32 & 125.41 \\
\hline 2 & 646.18 & 248.56 & 385.56 & 237.51 \\
\hline 3 & 2707.09 & 584.83 & 806.28 & 512.72 \\
\hline 4 & 223829.69 & 1389.10 & 2882.14 & 1116.85 \\
\hline 5 & abo & 11681.02 & 22532.75 & 3671.04 \\
\hline 6 & abo & 168217.65 & 373568.85 & 41344.03 \\
\hline 7 & abo & abo & abo & 458938.13 \\
\hline $20 \mathrm{~min}$ & $n=4$ & $n=6$ & $n=6$ & $n=7$ \\
\hline
\end{tabular}

Fig. 6. First experimental results for partial solvers run over benchmarks 


\begin{tabular}{|c|c|c|c|c|c|}
\hline \multirow{4}{*}{ Jurdzinski $[10, m]$} & $m$ & psol & psolB & psolQ & zlka \\
\hline & $10^{*} 2^{* *} 7$ & \begin{tabular}{l|l} 
abo & 1 \\
\end{tabular} & 179097.35 & abo & abo \\
\hline & $10^{*} 2^{* * 8} 8$ & \begin{tabular}{l|l} 
abo & 8 \\
\end{tabular} & 833509.48 & abo & abo \\
\hline & $20 \mathrm{~min}$ & $|n=560|$ & \begin{tabular}{l|l|l|}
$n=2890$ & $n$ \\
\end{tabular} & \begin{tabular}{l|l|l|}
$n=1120$ & $n$ \\
\end{tabular} & $=480$ \\
\hline \multirow{4}{*}{ Jurdzinski $[n, 10]$} & $n \|$ & psol & psolB & psolQ & zlka \\
\hline & \begin{tabular}{|l||l}
$10^{*} 2^{* *} 7$ & 3 \\
\end{tabular} & \begin{tabular}{l|l|}
308033.94 \\
\end{tabular} & 106453.86 & abo & abo \\
\hline & \begin{tabular}{|l||}
$10^{*} 2^{* *} 8$ \\
\end{tabular} & abo & 406621.65 & abo & abo \\
\hline & $20 \mathrm{~min} \|$ & $n=2420$ & \begin{tabular}{|l|l|}
$n=4380$ & $r$ \\
\end{tabular} & \begin{tabular}{l|l|l}
$n=1240$ & $n$ \\
\end{tabular} & $=140$ \\
\hline \multirow{4}{*}{$\operatorname{Jurdzinski}[n, n]$} & $n$ & psol & psolB & psolQ & zlka \\
\hline & $10^{*} 2^{* *} 3$ & 215118.70 & 23045.37 & 310665.53 & abo \\
\hline & $10^{*} 2^{* *} 4$ & abo & 403844.56 & abo & abo \\
\hline & $20 \mathrm{~min}$ & $n=110$ & $n=200$ & $n=100$ & $n=50$ \\
\hline
\end{tabular}

Fig. 7. Second experimental results run over Jurdzinski benchmarks

zlka was best for Model Checker Ladder and Elevator Verification, and about as good as psolB for Tower Of Hanoi. And psolQ was best for Recursive Ladder. Thus psol appears to perform worst across these benchmarks.

Solvers psolB and zlka seem to do about equally well for game types Clique, Ladder, Model Checker Ladder, and Tower Of Hanoi. But solver psolB appears to outperform zlka dramatically for game types Recursive Ladder, and Strategy Impr and is considerably better than zlka for Jurdzinski.

We think these results are encouraging and corroborate that partial solvers based on fatal attractors may be components of faster solvers for parity games.

\subsection{Number of Detected Fatal Attractors}

We also recorded the number of fatal attractors that were detected in runs of our partial solvers. One reason for doing this is to see whether game types have a typical number of dynamically detected fatal attractors that result in the complete solving of these games.

We report these findings for psol and psolB first: for Clique, Ladder, and Strategy Impr these games are solved by detecting two fatal attractors only; Model Checker Ladder was solved by detecting one fatal attractor. For the other game types psol and psolB behaved differently. For Recursive Ladder $[n]$, psolB requires $n=2^{k}$ fatal attractors whereas psolQ needs only $2^{k-2}$ fatal attractors. For Jurdzinski $[n, m]$, psolB detects $m n+1$ many fatal attractors, and psol removes $x$ edges where $x$ is about $n m / 2 \leq x \leq n m$, and detects slightly more than these $x$ fatal attractors. Finally, for Tower Of Hanoi $[n]$, psol requires the detection of $3^{n}$ fatal attractors whereas psolB solves these games with detecting two fatal attractors only.

We also counted the number of recursive calls for psolQ: it equals the number of fatal attractors detected by psolB for all game types except Recursive Ladder, where it is $2^{k-1}$ when $n$ equals $2^{k}$. 


\subsection{Experiments on Variants of Partial Solvers}

We performed additional experiments on variants of these partial solvers. Here, we report results and insights on two such variants. The first variant is one that modifies the definition of the monotone control predecessor to

$$
\begin{aligned}
\operatorname{mpre}_{p}(A, X, c)= & \left\{v \in V_{p} \mid((c(v) \% 2=p) \vee c(v) \geq c) \wedge v \cdot E \cap(A \cup X) \neq \emptyset\right\} \cup \\
& \left\{v \in V_{1-p} \mid((c(v) \% 2=p) \vee c(v) \geq c) \wedge v \cdot E \subseteq A \cup X\right\}
\end{aligned}
$$

The change is that the constraint $c(v) \geq c$ is weakened to a disjunction $(c(v) \% 2=$ $p) \vee(c(v) \geq c)$ so that it suffices if the color at node $v$ has parity $p$ even though it may be smaller than $c$. This implicitly changes the definition of the monotone attractor and so of all partial solvers that make use of this attractor; and it also impacts the computation of $A$ within psolQ. Yet, this change did not have a dramatic effect on our partial solvers. On our benchmarks, the change improved things slightly for psol and made it slightly worse for psolB and psolQ.

A second variant we studied was a version of psol that removes at most one edge in each iteration (as opposed to all edges as stated in Fig. 2). For games of type Ladder, e.g., this variant did much worse. But for game types Model Checker Ladder and Strategy Impr, this variant did much better. The partial solvers based on such variants and their combination are such that psolB (as defined in Figure 3) is still better across all benchmarks.

\subsection{Experiments on Random Games}

It is our belief that comparing the behavior of parity game solvers on random games does not give an impression of how these solvers perform on parity games in practice. However, evaluating our partial solvers over random games gives an indication of how often partial solvers completely solve random games, and of whether partial solvers can speed up complete solvers as preprocessors. So we generated 130,000 random games with the randomgame command of PGSolver.

Each game had between 10 and 500 nodes (average of 255). Each node $v$ had out-degree (i.e. the size of $v . E$ ) at least 1 , and at most $2,3,4$, or 5 - where this number was determined at random. These games contained no self-loops and no bound on the number of different colors. Then psolB solved $82 \%$ of these 130,000 random games completely. The average run-time over these 130, 000 games was $319 \mathrm{~ms}$ for psolB (which includes run-time of zlka on the residual game where applicable), whereas the full solver zlka took $505 \mathrm{~ms}$ on average. And only about 22,000 of these games (less than 17\%) were such that zlka solved them faster than the variant of zlka that used psolB as preprocessor.

\section{Conclusions}

We proposed a new approach to studying the problem of solving parity games: partial solvers as polynomial algorithms that correctly decide the winning status of some nodes and return a sub-game of nodes for which such status cannot be decided. We demonstrated the feasibility of this approach both in theory and 
in practice. Theoretically, we developed a new form of attractor that naturally lends itself to the design of such partial solvers; and we proved results about the computational complexity and semantic properties of these partial solvers. Practically, we showed through extensive experiments that these partial solvers can compete with extant solvers on benchmarks - both in terms of practical running times and in terms of precision in that our partial solvers completely solve such benchmark games.

In future work, we mean to study the descriptive complexity of the class of output games of a partial solver, for example of psolQ. We also want to research whether such output classes can be solved by algorithms that exploit invariants satisfied by these output classes. Furthermore, we mean to investigate whether classes of games characterized by structural properties of their game graphs can be solved completely by partial solvers. Such insights may connect our work to that of [3], where it is shown that certain classes of parity games that can be solved in PTIME are closed under operations such as the join of game graphs. Finally, we want to investigate whether and how partial solvers can be integrated into solver design patterns such as the one proposed in [5].

A technical report [7] accompanies this paper and contains - amongst other things - selected proofs, the pseudo-code of our version of Zielonka's algorithm, and further details on experimental results and their discussion.

\section{References}

1. Berwanger, D., Dawar, A., Hunter, P., Kreutzer, S.: DAG-Width and Parity Games. In: Durand, B., Thomas, W. (eds.) STACS 2006. LNCS, vol. 3884, pp. 524-536. Springer, Heidelberg (2006)

2. Chatterjee, K., Henzinger, M.: An $\mathrm{O}\left(n^{2}\right)$ time algorithm for alternating Büchi games. In: SODA, pp. 1386-1399 (2012)

3. Dittmann, C., Kreutzer, S., Tomescu, A.I.: Graph operations on parity games and polynomial-time algorithms. arXiv:1208.1640 (2012)

4. Emerson, E.A., Jutla, C.: Tree automata, $\mu$-calculus and determinacy. In: FOCS, pp. 368-377 (1991)

5. Friedmann, O., Lange, M.: Solving Parity Games in Practice. In: Liu, Z., Ravn, A.P. (eds.) ATVA 2009. LNCS, vol. 5799, pp. 182-196. Springer, Heidelberg (2009)

6. Friedmann, O., Lange, M.: The PGSolver Collection of Parity Game Solvers. Tech. report, Institut für Informatik, LMU Munich, Version 3 (February 2010)

7. Huth, M., Kuo, J.H., Piterman, N.: Fatal attractors in parity games. Tech. report no. 2013/1, Dep. of Computing, Imperial College London (January 2013) http://www.doc.ic.ac.uk/research/technicalreports/2013/DTR13-1.pdf

8. Jurdziński, M.: Small Progress Measures for Solving Parity Games. In: Reichel, H., Tison, S. (eds.) STACS 2000. LNCS, vol. 1770, pp. 290-301. Springer, Heidelberg (2000)

9. Jurdziński, M., Paterson, M., Zwick, U.: A deterministic subexponential algorithm for solving parity games. In: SODA, pp. 117-123. ACM/SIAM (2006)

10. Vöge, J., Jurdziński, M.: A Discrete Strategy Improvement Algorithm for Solving Parity Games. In: Emerson, E.A., Sistla, A.P. (eds.) CAV 2000. LNCS, vol. 1855, pp. 202-215. Springer, Heidelberg (2000)

11. Zielonka, W.: Infinite games on finitely coloured graphs with applications to automata on infinite trees. Theoretical Computer Science 200(1-2), 135-183 (1998) 